\title{
El Piano Complementario como herramienta de apoyo de los procesos de formación de los estudiantes de la Licenciatura en Música de la Universidad Pedagógica y Tecnológica de Colombia
}

\author{
The complementary piano as a tool to suport the processes of formation \\ of the students in the teaching music program in the UPTC
}

\begin{abstract}
Ángela Patricia Álvarez Castillo
Maestrante en Educación, Universidad Autónoma del Caribe, Especialista en Gerencia Educacional Colombia, Universidad Pedagógica y Tecnológica de Colombia angarez@gmail.com, Universidad Pedagógica y Tecnológica de Colombia, Avenida Central del Norte 39-115,
\end{abstract}

\section{Mónica María Tobo Mendivelso}

Maestrante en Educación, Universidad Autónoma del Caribe, Especialista en Pedagogía e Investigación en el Aula, Colombia, Universidad de la Sabana monicatobo@gmail.com, Universidad Pedagógica y Tecnológica de Colombia.

Para citar este artículo: Álvarez, A. \& Tobo, M. (2016). El Piano Complementario como herramienta de apoyo de los procesos de formación de los estudiantes de la Licenciatura en Música de la Universidad Pedagógica y Tecnológica de Colombia. Escenarios Vol 14 No1 p. p 130-146

DOI: http:/ / dx.doi.org/10.15665/esc.v14i1.880

Recibido: febrero 19 de 2016

Aprobado: marzo 31 de 2016

\begin{abstract}
RESUMEN
Este trabajo presenta una investigación terminada sobre la asignatura de Piano Complementario de la Licenciatura en Música de la UPTC, sistematiza las reflexiones en torno al desarrollo de la asignatura desde la creación del Programa en 1993. A partir de dichas reflexiones, así como de análisis de los contenidos curriculares de la asignatura en otros tres programas de licenciatura en música del país y del análisis de la percepción que docentes, estudiantes y egresados del programa tienen sobre la misma, plantea como resultado final una propuesta metodológica para el Piano Complementario que pretende responder de manera eficaz a las necesidades de los estudiantes del programa, que es el principal objetivo de esta investigación. El enfoque de esta investigación es cualitativo y el diseño es la micro-etnografía educativa. Este proyecto confirma a través de sus diferentes etapas la gran importancia que el Piano Complementario tiene para estudiantes y egresados del Programa y la necesidad de que dicha importancia se haga visible en la estructura curricular.
\end{abstract}

Palabras clave: Desarrollo de habilidades, formación profesional, competencias musicales, innovación curricular, formación musical 


\begin{abstract}
This work represents one finished investigation done in the te achine music program of the UPTC, It is about the complementary piano subject. It gather the main reflexions related to the development of the mentioned subject since the creation of the teaching music program, in 1993.

Taken those reflexions as the starting point, the contents given in another three paralel teaching music programs in Colombia and, analizing what teachers, studentes and graduated ex-students told about the complementary piano, this written work brings, as a result, a methodological porposal for the workshop of the complementary piano wich strives to answer in a succesfull way the identified needs of the program students, Grant this is the main goal of the investigation.

The perspective in this investigation is based on the quality of it's content and impact and it's design is given by the sistematic observation of a chosen group of students

This proyect states one more time the very important roll of the complementary piano in the profile of the students and graduated ex-students and, also stands for the idea that the piano complementary subject deserve and most remain in the studies program.
\end{abstract}

Keywords: Skills development, professional training, musical skills, curriculum innovation, musical training

\section{INTRODUCCIÓN}

La asignatura de Piano Complementario tiene un papel fundamental como herramienta de apropiación, entendimiento, asimilación y aplicación de diversos contenidos teóricos y pedagógico-musicales.

Dada la importancia que esta asignatura posee en los procesos de formación musical en sus diversas etapas, la casi totalidad de los programas de educación superior musical y pedagógico musical a nivel de pregrado la incluyen dentro de sus planes de estudio.

En la Licenciatura en Música de la Universidad Pedagógica y Tecnológica de Colombia la asignatura de Piano Complementario ha existido en los diversos planes de estudio desde la creación en 1993 y siempre ha tenido un carácter obligatorio. En la actualidad la asignatura está ubicada en los cuatro primeros semestres del plan de estudios.

Sin embargo, estudios preliminares a la realización de este proyecto han permitido determinar que la asignatura no está cumpliendo a cabalidad con sus objetivos, pues tanto docentes de asignaturas teóricas y pedagógicas como estudiantes manifiestan inconformismo frente a la conexión real que el Piano Complementario rea- liza con los aspectos teóricos y pedagógico-musicales desarrollados a lo largo de la carrera.

Desde hace algunos años algunos docentes de la asignatura en diversos lugares de Colombia y del mundo han replanteado han indo planteando nuevos enfoques para el Piano Complementario y han optado por crear materiales, métodos o cartillas orientadas específicamente a la enseñanza del piano complementario orientadas casi exclusivamente a la práctica del instrumento.

Dichas cartillas por lo general van acompañadas más de reflexiones empíricas surgidas desde la propia experiencia de sus creadores y no necesariamente son el resultado de un proceso de investigación en estricto sentido. Sin embargo, es necesario citar y tener en cuenta estos importantes materiales, pues a partir de ellos se puede establecer el tipo de orientación que busca dar cada uno de sus autores a la asignatura.

Este significativo incremento en la producción de nuevos materiales didácticos para Piano Complementario en el país está directamente relacionado con una inquietud cada vez mayor de los docentes por una investigación rigurosa sobre las necesidades locales en las diversas regiones en la que existen programas de formación musical y pedagógico musical.

Por otra parte, la creciente oferta de programas 
académicos de Maestría y Doctorado alrededor de diversas disciplinas musicales (Pedagogía del piano, educación musical, Interpretación instrumental, entre otras) ha llevado a un considerable incremento en las investigaciones y reflexiones sobre el Piano Complementario y en general sobre diversos aspectos de la pedagogía pianística en Colombia.

Es así como el trabajo de grado para optar al título de Magíster en Docencia Universitaria de Diego Palacios, titulado Currículo Pertinente para el Área de Piano Complementario del Programa de Licenciatura en Música de la Universidad de Nariño (2014), busca establecer las competencias necesarias para la formación de pianistas dentro de esta asignatura.

La Maestría en Pedagogía del Piano, ofertada por la Universidad Nacional de Colombia desde el año 2005 ha generado la realización de tesis de grado también relativas a diversas inquietudes de la enseñanza del instrumento.

Dentro de este programa de Maestría Esteban Bravo realizó su Trabajo de Grado titulado Enseñanza en grupo del piano para estudiantes de otras especialidades musicales (2009), en el cual busca responder a una pregunta fundamental ¿Cómo implementar un programa de piano colectivo para no pianistas, que sea atractivo a los involucrados más directos, profesores y estudiantes? Es por ello que propone una metodología en cuatro niveles para la enseñanza grupal del Piano Complementario, esto luego de analizar los escasos referentes escritos en particular sobre este tema.

Como reflejo de la producción investigativa de esta Maestría en Pedagogía del Piano encontramos también el Trabajo de Grado de Miyer Garvin (2011), titulado Guía metodológica para la enseñanza de algunas piezas para piano del maestro Luis Carlos Figueroa, en el cual cataloga en tres niveles de dificultad: iniciación, medio y avanzado las obras para piano de este compositor colombiano. Esto con el fin de brindar a los docentes de piano una guía que facilite la inclusión de dichas obras en los diversos momentos de formación pianística.

Por otra parte, como tesis previa para la obtención del título de Magíster en Pedagogía e Inves- tigación Musical de la Universidad de Cuenca (Ecuador), se encuentra el Diseño curricular para la cátedra de Piano Complementario en los conservatorios de música del Ecuador, una propuesta metodológica. En ella su autor Julio Bueno plantea un completo y detallado análisis sobre la historia de la cátedra de Piano Complementario y busca brindar varias estrategias didácticas para hacer esta asignatura más adecuada a las necesidades de los músicos ecuatorianos.

Vale la pena resaltar que todos estos trabajos para optar al título de Maestría articulan de manera muy acertada reflexiones teóricas que son el resultado de procesos de rigurosas investigaciones, con un componente práctico que busca brindar soluciones creativas a las problemáticas detectadas en la enseñanza pianística.

Todos los estudios mencionados se convierten en importantes referentes que permiten contextualizar el presente trabajo, que aborda diversas inquietudes en torno a la asignatura de Piano Complementario y busca fundamentalmente responder a la siguiente pregunta:

¿Cómo es el enfoque metodológico de la asignatura Piano Complementario le permite convertirse en una herramienta de apoyo de los procesos de formación de los estudiantes de la Licenciatura en Música de la UPTC?

Otras preguntas guían su desarrollo :¿Cuáles aspectos se han mantenido y cuáles se han modificado en la asignatura a lo largo de estos 23 años? ¿Qué opinión tienen estudiantes, egresados y docentes sobre la pertinencia e importancia de la asignatura? ¿Cómo se desarrolla la asignatura de Piano Complementario en otros programas de pregrado en música y en pedagogía musical a nivel nacional? De acuerdo con el perfil ocupacional de los egresados de la Licenciatura en Música de la UPTC ¿Qué habilidades se deben desarrollar dentro de la asignatura?

Por otra parte, se ha tenido en cuenta que "se reglamentan las condiciones de calidad para el otorgamiento y renovación del registro calificado de los programas académicos de licenciatura y los enfocados a la educación" (Decreto 2450 expedido el 17 de diciembre de 2015) y la Resolución 02041 del 3 de febrero de 2016 "Por la cual se establecen las características específicas de ca- 
lidad de los programas de Licenciatura para la obtención, renovación o modificación del registro calificado"

Lo anterior establece una reforma estructural a todas las Licenciaturas del país y que dicha reforma tiene un carácter obligatorio, se considera que es indispensable analizar las situaciones anteriormente mencionadas, pues de lo contrario se puede correr el riesgo de que la asignatura de Piano Complementario esté cada vez más descontextualizada de las necesidades reales de estudiantes, egresados y del medio en general en el cual ellos se desenvuelven.

Con miras a la implementación de dicha reforma en este trabajo se realiza en un primer momento un análisis de los aspectos más relevantes del Piano Complementario desde la creación del programa de Licenciatura hace 23 años. Para este análisis se tendrán en cuenta los contenidos programáticos de la asignatura, así como la opinión de egresados, docentes y estudiantes del Programa.

Se analizan también contenidos programáticos de Piano Complementario de otros 3 programas de Licenciatura en Música del país, con el fin de contrastarlos con los de la Licenciatura en Música.

Posteriormente se plantean cambios dentro de la asignatura que den solución a las problemáticas detectadas, con el fin de lograr que responda a las necesidades reales de los estudiantes de la Licenciatura. Para ello se establece un enfoque metodológico para la asignatura, lo cual es el objetivo general de este trabajo.

Esta investigación se considera no-experimental-transversal, teniendo en cuenta que en ella no se manipulan de forma intencional las variables seleccionadas, ni se ejerce ningún tipo de control sobre las mismas. Además se centra en analizar cuál es el nivel o estado de una o diversas variables en un momento dado y de cuál es la relación que existe entre ellas en un momento determinado.

El diseño metodológico seleccionado para desarrollar esta investigación es el cualitativo de micro-etnografía educativa. De acuerdo con Murillo y Martínez en su libro Métodos de in- vestigación educativa (2010), se considera que el diseño etnográfico, "Es quizá el método más conocido y utilizado en el campo educativo para analizar la práctica docente, describirla desde el punto de vista de las personas que en ella participan y aproximarse a una situación social". En particular la etnografía educativa se adapta al planteamiento del problema ya que "se centra en descubrir lo que allí acontece cotidianamente a base de aportar datos significativos, de la forma más descriptiva posible, para luego interpretarlos y comprender e intervenir adecuadamente en esa realidad particular de cada aula." (2010)

Un aspecto importante que llama la atención sobre la etnografía educativa y que también se adapta a los propósitos de esta investigación es que no se queda en la simple descripción de la asignatura de Piano Complementario, sino que propende por sugerir tras el análisis alternativas que solucionen las problemáticas detectadas.

Perspectiva Teórica La búsqueda y análisis de referentes teóricos se realizó, con la intención de tener una base relacionada con la gestión y administración curricular, el desarrollo cognitivo y la didáctica y pedagogía de la música. A continuación se desglosa cada aspecto antes mencionado:

Este proyecto investigativo, si bien es de tipo pedagógico tiene un componente curricular fundamental en lo relacionado al análisis, diagnóstico, diseño e innovación de contenidos curriculares de la asignatura Piano Complementario de la Licenciatura en Música UPTC. Las cuatro reestructuraciones de los planes de estudio en la Licenciatura en Música UPTC, han obligado a replantear los objetivos del programa, la misión, la visión, el perfil, la malla o estructura curricular, la filosofía y el modelo pedagógico. Estas modificaciones que buscaron principalmente ser coherentes con el contexto social y cultural del medio y elevar los niveles de calidad de la educación, deben tener un impacto sustancial en lo referente a los contenidos programáticos de todas las asignaturas y su implementación, esto si realmente se quieren logran cambios significativos en la manera como se desarrolla cualquier programa académico, es así como:

Si el currículo llega a los profesores que 
han de aplicarlo como resultado de la elaboración teórica y aislada de un grupo selecto de expertos, entonces cada uno de los profesores lo adecuará a sus propias vivencias, en correspondencia con su formación pedagógica y profesional, y el resultado seguirá siendo el mismo que antes en líneas generales. (Horruitiner Silva, 2006: 2)

En tal sentido, las instituciones de educación superior de los países latinoamericanos, están obligadas a desarrollar currículos innovadores que respondan a las actuales exigencias competitivas a nivel educativo, dadas por la globalización y la internacionalización de la educación. “En las universidades de nuestra región se están haciendo grandes esfuerzos para adecuarlas a la necesidad de producir -de manera permanentesaberes que contribuyan a crear las condiciones de un desarrollo sustentable para todos y cada uno de los sectores de la sociedad. Innovar ya no puede ser un accidente feliz, es una obligación constante." (Medina Cuevas \& Guzmán Hernández, 2011:19). La innovación curricular que se propone en esta investigación, no solo se plantea desde el trabajo de campo y análisis de documentos relacionados con el mismo, sino desde el enfoque teórico conceptual acerca de las consideraciones fundamentales para realizar una transformación curricular. “(...) la innovación en el campo curricular debe considerar cuatro grandes ámbitos para su formulación: a) el ámbito territorial, b) el ámbito económico, c) el ámbito político y d) el ámbito sociocultural," (Medina Cuevas \& Guzmán Hernández, 2011: 21).

A partir de los cuatros ámbitos anteriormente nombrados, que se proponen aplicar de una manera general para las reestructuraciones de planes de estudio, se pueden fundamentar las transformaciones en un nivel más particular como es la modificación, ajuste y propuesta de contenidos curriculares de una asignatura.

Currículo Oculto Para realizar un análisis veraz y real de los contenidos curriculares de una asignatura, es fundamental observar si hay coherencia entre lo registrado en el documento de contenidos y las actividades que el docente desarrolla dentro del aula.

Es ahí, en el aula, en la actividad práctica del profesor con sus estudiantes, donde se decide realmente la efectividad de cualquier proceso de transformación curricular. En cada profesor universitario reside, como resultado de su labor académica, un currículo oculto que lo guía, que lo conduce a hacer las cosas en correspondencia con su experiencia precedente y que en última instancia, con mucha frecuencia, refleja la forma en que a él le fueron impartidos esos contenidos.(Horruitiner Silva, 2006)

De esta manera, el currículo oculto se convierte en un indicador de la implementación de los contenidos en el aula y si realmente se están cumpliendo o no. Al respecto, Cantón Mayo y Vargas (2009) señalan que "en el aula siempre existe un currículo prescrito y un currículo real, el primero se da como una intensión, idea o plan de lo que desea la institución y el segundo como lo que se concretiza en el aula". Por otra parte, al analizar el currículo oculto se pueden detectar aspectos valorativos, desarrollo de destrezas y actitudes que en el papel no son explícitos.

Según Carrillo Siles (2009), podemos entender el currículo oculto como aquel que hace referencia a los conocimientos, destrezas, valores, actitudes, normas que se adquieren en los procesos de enseñanza aprendizaje y, en general, en todas las interacciones que se dan cotidianamente en el aula y en la escuela, pero que no llegan a explicitarse como metas educativas a lograr de una manera intencionada.

Psicología de la música El campo de la psicología de la música, tiene que ver con todos los procesos cognitivos que se gestan a partir del desarrollo del pensamiento musical y que se conectan en su mayoría de veces con la adquisición de habilidades motoras. En el caso particular del piano complementario que es la asignatura estudiada en esta investigación, el desarrollo de habilidades y destrezas relacionadas con la interpretación del piano, debe estudiarse y comprenderse con la finalidad de proponer contenidos que se enfoquen en la adquisición de las habilidades necesarias. En primer lugar, debemos caracterizar el término de habilidad para entender luego como enseñarla para que pueda ser adquirida. En este sentido, el reconocido 
psicólogo de la música John Sloboda (Gellatly, 1997:36) establece cinco características principales de la habilidad: Fluidez, rapidez, automaticidad, simultaneidad y conocimiento. Por otro lado Davison y Scripp (1992), hablan de tres etapas para la adquisición de una habilidad de ejecución musical:

La producción, la percepción y la reflexión, esto es (...) a) "los procedimientos expresados en la acción misma, b)la percepción en la acción como resultado de observar la expresión y c) la reflexión en la ejecución, donde la toma de decisiones se produce por la influencia de los eventos que van surgiendo en el momento" (Tuñez\& Zabala 2007:48).

Brian More citado por (Boardman,1989), sobre los procesos de pensamiento musical, habla de tres características principales del pensamiento musical que está relacionado directamente con la habilidad: contenido, contexto y calidad. Estas tres enmarcadas dentro de la organización y fundamentación del conocimiento. Entrando ahora a la adquisición de las habilidades, Sloboda (Gellatly, 1997:51) menciona tres factores que condicionan dicha adquisición: Condiciones trasmitidas genéticamente, características físicas y factores de disposición y motivación. Sin embargo ubica a la práctica como el factor más importante en la adquisición de una habilidad. "No resultará sorprendente enterarnos que el factor simple más importante que los psicólogos conocen es la práctica. De la misma manera la práctica es el ingrediente que más destacan los entrenadores deportivos y los maestros de piano." (Gellatly, 1997:52). Es así como la transferencia de aprendizaje se vuelve fundamental en la adquisición de una habilidad. "Mientras más experiencia se tenga con el aprendizaje disponible, más oportunidad y probabilidad habrá de que ocurra la respuesta correcta en la conducta." (Regelski, 1980).

Fundamentos pedagógicos Todo aprendizaje a nivel universitario, debe estar articulado con los conocimientos previos con que el estudiante llega al aula. Específicamente en la asignatura de Piano Complementario en la UPTC, en donde los materiales actualmente utilizados muestran un interés de la institución por articular las músicas populares a la academia, es decir, contextualizar los contenidos a las necesidades de la región, es fundamental conectar las ideas, expectativas y conocimientos que los estudiantes traen con las metas y propósitos de la clase.

En tal sentido, el aprendizaje significativo, teoría planteada por Ausbel (1976) donde "los contenidos son relacionados de modo no arbitrario y sustancial con lo que el alumno ya sabe", se ajusta a lo que se pretende con la asignatura en cuestión. El modelo pedagógico constructivista, sigue la idea del aprendizaje significativo en el sentido de que "es la idea que mantiene el individuo-, en los aspectos cognitivos y sociales del comportamiento y en los afectivos- (...) es una construcción propia que se va produciendo día a día como resultado de la interacción entre esos dos factores." (Carretero, 2000:39).

Siguiendo esta idea, Ausbel insistía en que aprender es sinónimo de comprender. Por ello, lo que se comprenderá será lo que se aprenderá y recordará mejor porque quedará integrado en nuestra estructura de conocimientos" (Carretero, 1997:44). Por otra parte, "Piaget sostiene que, a partir de unas capacidades generales con las que se nace, los sujetos van construyendo su inteligencia y su conocimiento sobre la realidad." (Delvat, 2001: 354)

El PAE del programa de Licenciatura en Música de la UPTC, ratifica estos fundamentos pedagógicos y plantea el aprendizaje autónomo, significativo y colaborativo como ejes fundamentales de la enseñanza musical. Así mismo, toma como modelo pedagógico el constructivismo donde (...) "el estudiante es el centro y objeto del proceso educativo, es dinámico, creativo, innovador y constructor de su propio conocimiento a partir del contacto con el medio, gestor de sus propios procesos" (PAE, 2010).

Justificación El proyecto se plantea por el interés de sistematizar inquietudes surgidas a partir de la práctica pedagógica cotidiana dentro de la Licenciatura en Música de la UPTC. Además surge de la profunda necesidad de comprender desde una perspectiva integral la realidad del desarrollo de la asignatura de Piano Complementario, con el fin de plantear a futuro un enfoque metodológico que responda plenamente a las necesi- 
dades del medio.

No existen investigaciones que aborden el tema específico del Piano Complementario en la Licenciatura en Música de la UPTC, lo que sin duda redunda en un desconocimiento por parte de toda la comunidad que la integra sobre el impacto real que la asignatura tiene dentro y fuera del programa.

Es por ello que se pretende que los resultados del proyecto permitan una articulación cada vez mayor entre la asignatura de Piano Complementario y las necesidades de los estudiantes de la Licenciatura en Música de la UPTC.

Si bien la Licenciatura en Música de la Universidad Pedagógica y Tecnológica de Colombia tiene 23 años de funcionamiento, la reflexión sobre el contexto social y cultural en el que se encuentra inmersa es un tema que tan solo en los últimos años ha sido objeto de investigación.

Los directos beneficiados con los resultados de esta investigación serán tanto docentes como estudiantes de la Licenciatura en Música, pues en primer lugar una vez la UPTC determine la realización e implementación de la reforma prevista por el Ministerio de Educación Nacional, el programa ya contará con una perspectiva mucho más amplia sobre cómo debe plantearse la asignatura de Piano Complementario. Esto sin duda hará que los estudiantes se apropien del piano como una verdadera herramienta de apoyo en su proceso de formación pedagógico-musical y que los docentes de asignaturas teóricas y pedagógicas lleven desarrollen sus procesos de enseñanza-aprendizaje de una forma mucho más fluida.

$\mathrm{Su}$ incidencia teórico-práctica, busca caracterizar la asignatura de Piano Complementario dentro de la Licenciatura en Música de la UPTC, contrastándola además con el Piano Complementario que se dicta en otras universidades del país. De otra parte se evidencia una clara aplicación práctica, pues plantea unos nuevos contenidos programáticos para la asignatura de Piano Complementario mucho más acordes con las necesidades reales de los estudiantes que cursan el programa.

Se determinó si realmente el piano complemen- tario, ha cumplido su función básica de herramienta que apoya los procesos de formación teórica y pedagógica de los estudiantes. Luego de este análisis de la asignatura de Piano Complementario, desde su creación hasta el día de hoy, se proyectan unos contenidos programáticos para la asignatura que respondan de manera cada vez más eficaz a las necesidades de los estudiantes del Programa y que conviertan al Piano Complementario en una herramienta de apoyo en sus propios procesos de formación educativa.

\section{Metodología}

Respecto al tipo de investigación teniendo en cuenta que esta investigación parte de establecer el desarrollo histórico que ha tenido la asignatura de Piano Complementario a lo largo de los 22 años de creación del Programa de Licenciatura en Música de la UPTC, se considera que hay un enfoque retrospectivo.

A partir de dicho análisis, de la comparación con la forma como se implementa la asignatura en otros programas de pregrado de Licenciatura en Música del país y de las apreciaciones de docentes, egresados y estudiantes de la Licenciatura en Música de la UPTC, se pretende proponer unos nuevos contenidos programáticos para la asignatura, de forma que respondan a las necesidades del medio. Por lo tanto esta investigación tiene también un componente de tipo prospectivo.

El diseño seleccionado para desarrollar esta investigación es el cualitativo de micro-etnografía educativo. Consideramos que la idea de investigación puede ser ampliamente explorada a partir de este diseño por varias razones Es no-experimental-transversal, teniendo en cuenta que en ella no se manipulan de forma intencional las variables seleccionadas, ni se ejerce ningún tipo de control sobre las mismas. Además se centra en analizar cuál es el nivel o estado de una o diversas categorías en un momento dado y de cuál es la relación que existe entre ellas en un momento determinado.

De acuerdo con Murillo y Martínez (2010), se considera que el diseño etnográfico, "Es quizá el método más conocido y utilizado en el campo educativo para analizar la práctica docente, 
describirla desde el punto de vista de las personas que en ella participan y aproximarse a una situación social". (2010) En particular la etnografía educativa se adapta al planteamiento del problema ya que "se centra en descubrir lo que allí acontece cotidianamente a base de aportar datos significativos, de la forma más descriptiva posible, para luego interpretarlos y comprender e intervenir adecuadamente en esa realidad particular de cada aula." (2010)

Los recursos con los que se cuenta para la recolección de datos son amplios, suficientes y de fácil acceso para las investigadoras: entrevistas a egresados, a estudiantes y a docentes, documentos curriculares, contenidos programáticos, videos de conciertos y de materiales desarrollados en la clase, con participación de las investigadoras.

Se establece una categoría central alrededor de la cual se estructuran las demás: El piano complementario como herramienta. A partir de esta categoría surgen otras como: articulación con los contenidos programáticos, articulación con los planes de estudio, percepción de los participantes, entre otras. Analizar la asignatura de piano complementario desde la etnografía educativa, sistematiza de manera rigurosa observaciones informales que se han desarrollado a partir de la experiencia misma en la docencia de la asignatura.
En la tabla 1.0 se muestra la relación entre el tipo, el propósito del diseño con el proceder investigativo.

La investigaciones de tipo teórico, por cuanto busca hacer una revisión crítica y comparando distintas perspectivas del Piano Complementario. De acuerdo a la forma como está planteada la investigación, el método transita por diferentes momentos:

Un primer momento analítico-sintético: En el cual se analizan por separado las diversas categorías planteadas en el proyecto, tratando así de comprender sus partes estructurales más importantes, para posteriormente establecer algunas generalizaciones a partir de dicho análisis.

Un segundo momento histórico- lógico: En esta etapa se brinda una contextualización histórica de la asignatura del Piano Complementario en la Licenciatura en Música de la UPTC, para así determinar de qué manera se han desarrollado sus aspectos estructurales a través del tiempo.

Un tercer momento hipotético-deductivo: A partir de los análisis desarrollados en los dos primeros momentos se establecen unas hipótesis tanto de lo que ha sido la asignatura de Piano Complementario en la Licenciatura en Música de la UPTC a través de la historia, como de las que pueden ser las actuales necesidades de quienes cursan la asignatura.

Tabla 1.0 Tipo, propósito y proceder investigativo

\begin{tabular}{|c|c|c|}
\hline Tipo de Diseño & Propósito del diseño & Proceder Investigativo \\
\hline Micro-etnografía educativa & $\begin{array}{l}\text { Obtener información empírica } \\
\text { en el espacio en donde se } \\
\text { desenvuelven los } \\
\text { acontecimientos estudiados, } \\
\text { "documentar lo no } \\
\text { documentado", permitiendo así } \\
\text { una elaboración cualitativa del } \\
\text { contexto escolar estudiado; sus } \\
\text { resultados en un texto que } \\
\text { describe densamente la } \\
\text { especifidad del lugar }\end{array}$ & $\begin{array}{l}\text { 1. Selección del diseño } \\
\text { 2. La determinación de las } \\
\text { técnicas } \\
\text { 3. El acceso al ámbito de } \\
\text { investigación } \\
\text { 4. La selección de los } \\
\text { informantes. } \\
\text { 5. La recogida de datos y la } \\
\text { determinación de la duración } \\
\text { de la estancia en el escenario. } \\
\text { 6. El procesamiento de la } \\
\text { información recogida. } \\
\text { 7. La elaboración del informe. }\end{array}$ \\
\hline
\end{tabular}

Fuente: Trabajo de investigación maestría en educación UAC (Tobo,M \& Alvarez, A, 2016-2 ) 
Finalmente pasa por el enfoque de sistema: Esto teniendo en cuenta que el principal objetivo de este tipo de enfoque es la solución de un problema concreto y real. Además la investigación abarca sus etapas esenciales: 1) El desarrollo de conceptos y lineamientos para estudiar la realidad como un sistema. 2) El desarrollo de esquemas metodológicos para orientar el proceso de solución de problemas. 3) El desarrollo de un modelo para apoyar la toma de decisiones, así como para obtener y analizar la información requerida.

El método utilizado en esta investigación es inductivo en un primer momento, por cuanto se busca analizar todo el problema para describir sus elementos constitutivos y de qué manera, estos influyen en el desarrollo del problema. Posteriormente y teniendo en cuenta que la propuesta se va a aplicar en el Programa de Licenciatura en Música de la UPTC y que además se va a recurrir al criterio de especialistas, se considera que la investigación es también deductiva en su última etapa.

Respecto a la población y muestra del proyecto, se contó con una muestra significativa de estudiantes y/o egresados que hayan cursado la asignatura a lo largo de estos 23 años de creación del programa. Por ello se aplicó una encuesta a una muestra aleatoria de egresados que cursaron diferentes planes de estudios.

Por otra parte se aplicaron dos tipos de encuesta para los estudiantes: una para aquellos que aún cursan la asignatura de Piano Complementario y otra para quienes ya completaron los 4 niveles de la misma.

Se entrevistó a los dos docentes que actualmente dictan la asignatura de Piano Complementario.

Para el desarrollo de esta investigación se consultaron fuentes primarias como los Contenidos Curriculares de la asignatura y el Proyecto Académico Educativo de la Licenciatura en Música de la UPTC. Se consultaron también fuentes secundarias como: Memorias de Eventos que den cuenta de temáticas pertinentes a este proyecto de investigación.

Las técnicas empleadas fueron: Observación a grupos focales, entrevistas, encuestas y análisis y revisión de textos.
El proyecto está estructurado en 4 grandes etapas que tienen que ver con recopilar, procesar, presentar y analizar la información.

- Recopilación de información textual

- Observación de la muestra de población seleccionada

- Análisis de los resultados obtenidos

- Propuesta metodológica para la asignatura

\section{Resultados y Análisis.}

Luego de hacer una búsqueda de antecedentes y de referentes, y de realizar un análisis exhaustivo de documentos, encuestas y entrevistas, la investigación arroja resultados contundentes respecto al estado actual de la asignatura de piano complementario y la necesidad de realizar ajustes y modificaciones a los contenidos curriculares de dicha asignatura. Por una parte, el análisis comparativo de los contenidos de la UPTC a lo largo de los 22 años de funcionamiento de la Licenciatura en Música, evidencia cambios importantes en cuanto a objetivos, número de semestres, definición y enfoque metodológico.

En una primera etapa (1993 a 2002) los objetivos generales se orientan a una apropiación técnica, de lectura musical, armonía, expresividad y un manejo dinámico del piano. Por su parte los contenidos de 2003 a 2009 en dicho apartado se hacen énfasis en el piano como herramienta y se menciona la necesidad de articularlo a la aplicación real en el medio. A partir del 2010 llama la atención que los contenidos programáticos no contemplan el ítem Objetivos. Sin embargo, en las unidades programáticas se evidencia que se propende por un desarrollo integral de diversas competencias. Así mismo se busca que haya articulación con el enfoque constructivista que tiene el Proyecto Educativo Institucional de la Licenciatura en Música.

En cuanto el número de niveles, es notoria la reducción que ha experimentado la asignatura a lo largo de la carrera, pues en un primer momento eran 8 niveles y posteriormente se redujeron a 4. Este hecho sin duda afecta negativamente el desarrollo de la asignatura, si se tiene en cuenta que la mayor parte de los estudiantes ingresan al 
Gráfico 1.0 Importancia del piano en la formación musical

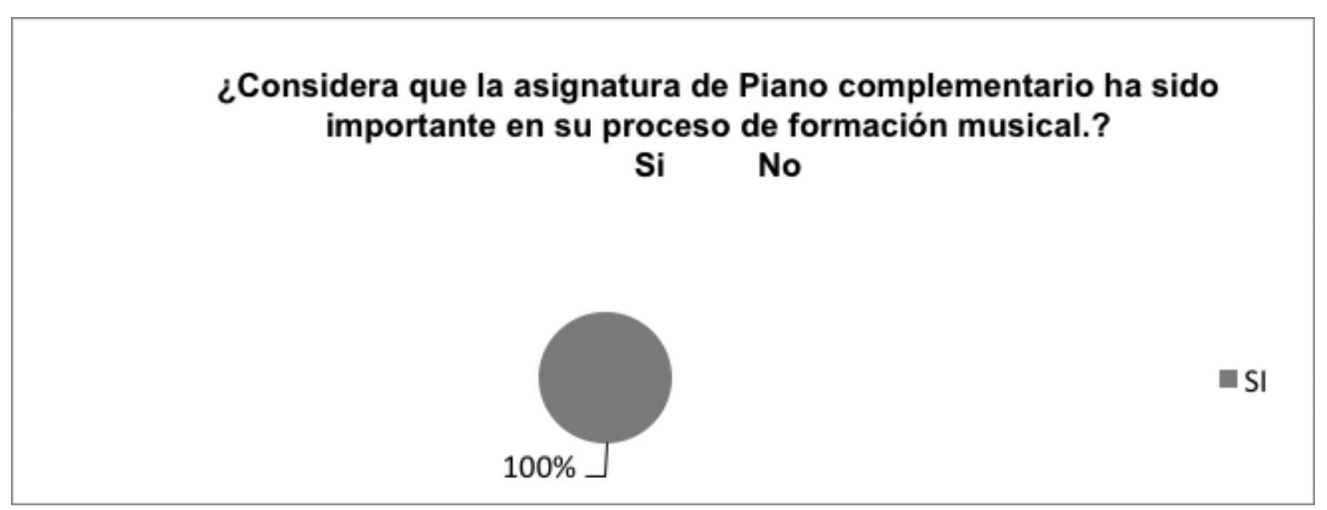

Fuente: Trabajo de investigación maestría en educación UAC (Tobo,M \& Alvarez, A, 2016-2)

programa de Licenciatura en Música sin conocimientos pianísticos.

En un primer momento los estudiantes cursaban a lo largo de todos los semestres una hora individual de clase. A partir del 2004, se empezó a trabajar una hora semanal de clase en grupos de 3 estudiantes. Este trabajo grupal favorece el aprendizaje colaborativo, pues el espacio de la clase favorece que se pueda aprender a partir de los aciertos y errores de los otros estudiantes. Sin embargo los estudiantes tienen ritmos diferentes en el aprendizaje y dominio del piano, el seguimiento de dichos ritmos es más complejo de desarrollar mediante clases colectivas.

La asignatura de Piano Complementario siempre ha sido concebida como una herramienta de apoyo a los procesos de formación musical, teórica y pedagógica de los estudiantes, pero solo en los dos últimos momentos analizados (2003 a 2009 y 2010 a 2016) se menciona la necesidad de relacionarla de manera directa con las demás asignaturas que integran el currículo y contextualizar los materiales y habilidades que se desarrollan de acuerdo a las necesidades reales a las que se enfrentan los egresados. Por último, el enfoque metodológico en los dos primeros momentos analizados es bastante general y no se menciona de qué manera se trabajarán cada una de las unidades temáticas.

La metodología se centra exclusivamente en el desarrollo técnico, la lectura y el conocimiento del repertorio. Esta metodología empleada guarda una gran similitud a la empleada comúnmente en la asignatura de Instrumento Princi- pal. Los materiales abordados pertenecen en su totalidad a la tradición musical clásica europea, exceptuando la inclusión de un libro de piezas fáciles de jazz en el 2003.

En el último momento (desde el 2010 hasta la actualidad) se hace explícito el tipo de enfoque pedagógico que sustenta la asignatura (constructivista) y se aclara además la forma de trabajo con la que se desarrollarán las diversas actividades previstas. Entre los materiales más usados, está una cartilla diseñada por una de las docentes del programa de Licenciatura en Música de la UPTC que ha unificado la manera como los docentes desarrollan la clase y en la que se incluyen ejercicios basados en ritmos colombianos y latinoamericanos.

Por otra parte, el diagnóstico actual realizado a la asignatura de Piano Complementario a través de encuestas y entrevistas a los agentes implicados (estudiantes, docentes, egresados) y del análisis del PAE (plan académico educativo) del programa, muestra que efectivamente la percepción que se tiene de la asignatura es que es fundamental para la formación musical y pedagógica de un Licenciado en Música (Gráfico 1.0).

El número de niveles es adecuado aunque los profesores recomiendan que se eleve a 5 o 6 niveles. En cuanto al número de estudiantes, manifiestan que es adecuado pero sería mucho mejor si la intensidad en créditos aumentara. Las habilidades que se desarrollan en la clase (técnica, acompañamiento, armonización, lectura de partituras, lectura de cifrado e improvisación) son pertinentes para el desempeño de un Licenciado en música (Gráfico 2.0). 
Gráfico 2.0 Importancia de las temáticas desarrolladas en el Piano Complementario

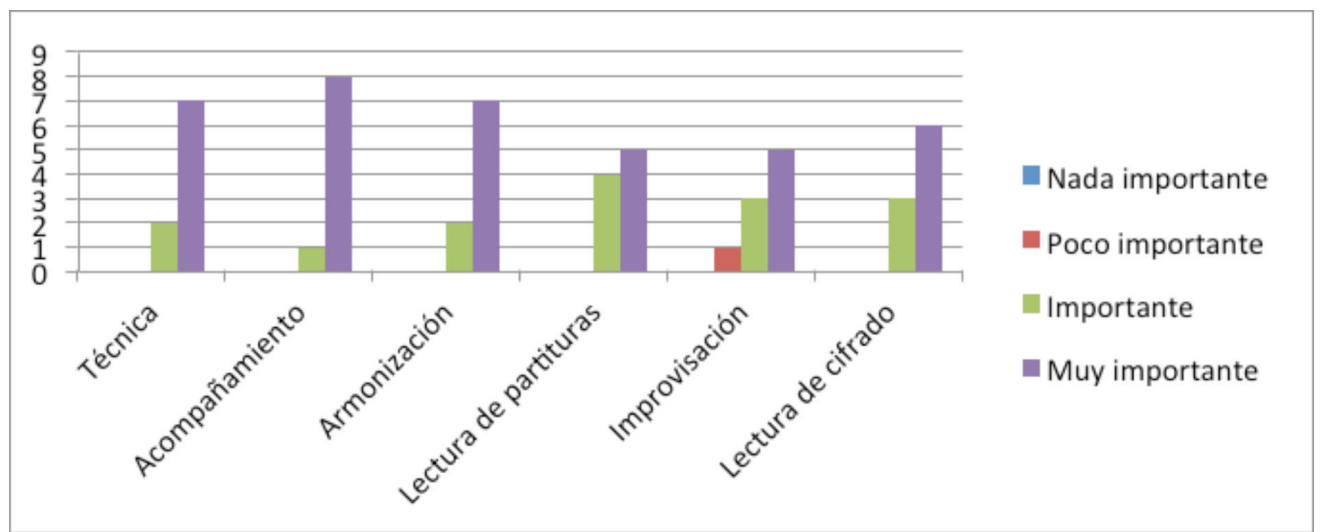

Fuente: Trabajo de investigación maestría en educación UAC (Tobo,M \& Alvarez, A, 2016-2)

La metodología utilizada actualmente es adecuada (Gráfico 3.0), sin embargo los estudiantes sugieren que se realice transferencia de aprendizaje en contextos más reales y prácticos. También manifiestan buscar más materiales a parte de la cartilla que se utiliza e incluir el estudio más profundo de la música colombiana (Gráfico 4.0). La articulación de esta asignatura con otras del pensum, es muy importante según la opinión de todos los encuestados. Las asignaturas donde más se ve reflejado el piano como herramienta de apoyo es en Educación auditiva y en Armonía (Gráfico 5.0).

Sin embargo, los docentes manifiestan que se debe ayudar a los estudiantes a realizar la transferencia de conocimientos entre asignaturas por medio de estrategias desarrolladas conjuntamente por los docentes. Así mismo, es importante sincronizar contenidos, materiales y léxico en- tre el piano complementario y asignaturas como, educación auditiva, armonía e instrumento principal. Igualmente importante es realizar la articulación de la asignatura piano complementario con el PAE de la Licenciatura en Música.

En los contenidos actuales de la asignatura de Piano Complementario se puede apreciar que los tres ejes transversales que estipula el PAE (audición, interpretación y creación) están presentes a través de todos los niveles, pues la asignatura contempla la interpretación de obras musicales y presentaciones en público, además de ello el componente armónico de las canciones populares que los estudiantes seleccionen debe ser transcrito por ellos mismos, lo que sin duda implica el desarrollo de la audición. Por otra parte, en el último nivel de la asignatura los estudiantes deben realizar pequeñas improvisaciones a partir de esquemas armónicos presentados en el

Gráfico 3.0 Percepción de los aspectos desarrollados en clase

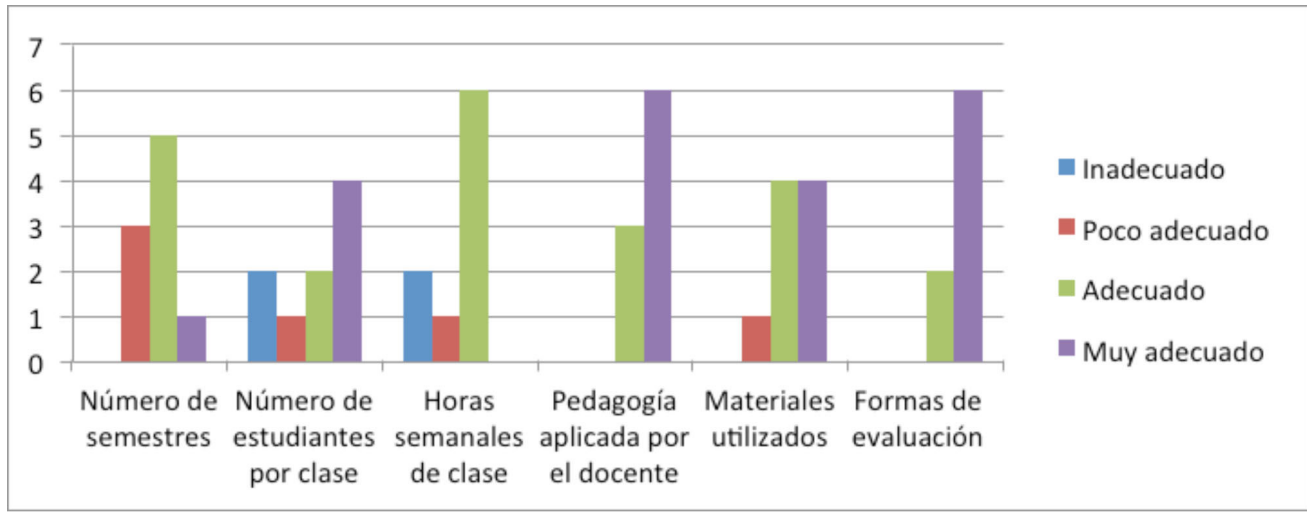

Fuente: Trabajo de investigación maestría en educación UAC (Tobo,M \& Alvarez, A, 2016-2) 
Gráfico 4.0 Modificaciones a realizar

¿Le realizaria modificaciones a los contenidos programáticos de piano complementario que usted cursó?

Si No

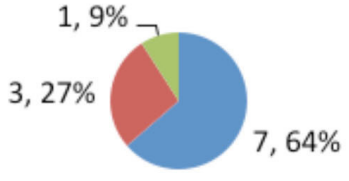

- Herramienta funcional y enfóque pedagógico

- Uso de musicas colombianas y populares

Fuente: Trabajo de investigación maestría en educación UAC (Tobo,M \& Alvarez, A, 2016-2)

Gráfico 5.0 Articulación con Asignatura

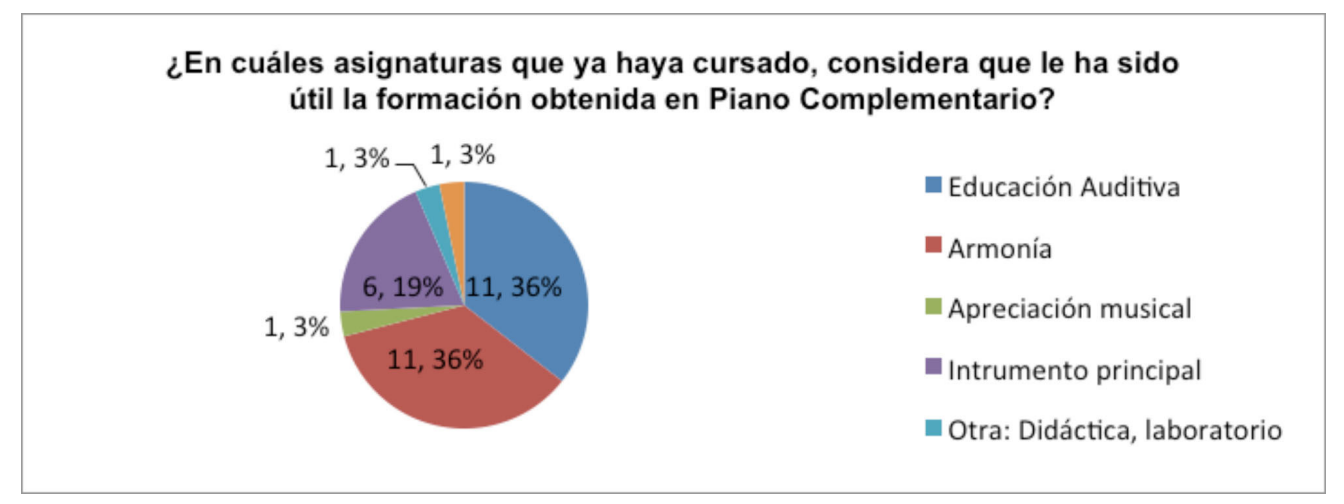

Fuente: Trabajo de investigación maestría en educación UAC (Tobo,M \& Alvarez, A, 2016-2)

texto guía, con lo cual se promueve la creación.

El perfil profesional y ocupacional de la Licenciatura se centra en formar un Músico educador capaz de desarrollar procesos de educación musical en diferentes contextos. La asignatura de Piano Complementario en la actualidad tiene un componente de desarrollo pedagógico pues comprende en los diversos niveles unidades temáticas orientadas a la armonización y acompañamiento de canciones infantiles, así como de repertorios populares que son escuchados de forma permanente por niños y jóvenes.

Por último, los aprendizajes promovidos en el PAE de la Licenciatura son el autónomo, el significativo y el colaborativo. Sin duda cualquier proceso de formación en un instrumento musical implica un trabajo individual de cada uno de los estudiantes, que tiene que ver con la apropiación de aspectos motrices, de lectura música y de interpretación. La idea de trabajar con músicas populares que son del agrado de los estudiantes está directamente relacionada con que el aprendizaje del piano sea significativo para ellos y represente algo con lo que se pueden identificar.

Tomando como punto de partida los aspectos relevantes que marcan las tendencias curriculares actuales, se analizaron tres programas de licenciatura en Música (Universidad Pedagógica Nacional, Universidad de Pamplona y UPTC) encontrando lo siguiente: 1) Respecto a la inclusión de nuevas Tecnologías, los contenidos curriculares de la UPTC evidencian el manejo herramientas tecnológicas como el correo electrónico y las plataformas de video como Youtube y Wathsapp. Por el contrario, las otras dos universidades no contemplan dentro de sus estrategias metodológicas la utilización de la tecnología para sus clases de piano complementario. 2) Respecto al enfoque constructivista, al ser 
una asignatura que comúnmente se trabaja de manera personalizada, aunque no se evidencia en los contenidos de la UPN, la metodología está casi siempre relacionada con el ritmo de aprendizaje de cada estudiante. Esto quiere decir que el docente debe tener en cuenta los conocimientos que el estudiante trae previamente y como su aprendizaje se adquiere con base a estos. El aprendizaje colaborativo, se ve reflejado en el montaje de repertorio de manera grupal, como montaje a cuatro manos (UPN) y montaje de repertorio popular con agrupaciones (UPTC).

En cuanto a la manera como se transmiten los conocimientos, en la UPTC se muestra un trabajo por recepción verbal, aprendizaje por descubrimiento y transferencia de conocimientos. En la Universidad de Pamplona, los contenidos se abordan de manera progresiva, de lo más básico a lo más complejo. Así mismo presentan un trabajo teórico apoyado por la práctica directa en el instrumento.

3) En cuanto al fortalecimiento de la identidad cultural y musical, los tres programas presentan unos contenidos y bibliografía que incluye música folklórica y tradicional colombiana, así mismo se especifica el trabajo con música de diferentes géneros y patrones de acompañamiento rítmico armónico dados por músicas diversas. Allí se manifiesta la preocupación de tener en cuenta los intereses musicales de los estudiantes y las necesidades culturales y sociales del contexto regional y nacional. 4) En este aspecto de la mirada académica del piano como herramienta armónica, al analizar tanto los objetivos como metodología y contenidos, los tres programas de Licenciatura en Música, pretenden desarrollar habilidades técnicas, teóricas, armónicas e interpretativas en el piano como instrumento armónico, donde el estudiante pueda adquirir las herramientas necesarias para su buen desempeño como estudiante y como futuro docente en música. La mirada funcional del instrumento es evidente y se trabajan materiales que permiten adquirir habilidades de acompañamiento rítmico-armónico como por ejemplo los patrones rítmicos el montaje de canciones infantiles y populares, habilidades de lectura de cifrado americano, habilidades de improvisación, habilidades de armonización de melodías utilizando progresiones armónicas y en general se observa que la concepción del piano como instrumento de apoyo a los procesos cognitivos de los estudiantes ha ido cambiando en la última década gracias a los estudios y reflexiones investigativas que se han realizado en toda Iberoamérica.

\section{Propuesta Metodológica para la asignatura de Piano Complementario de la Licenciatura en Música}

A partir del análisis de los documentos y los instrumentos aplicados, se presentan de manera sintética, los aspectos estructurales de la nueva propuesta metodológica para la asignatura de Piano Complementario de la Licenciatura en Música de la UPTC. La propuesta, es el resultado de los análisis previos y busca responder de una forma cada vez más eficaz a las necesidades del medio.

El nombre de la asignatura "Piano complementario" se mantiene como está, ya que es un instrumento de apoyo a los procesos de formación musical y pedagógica de los estudiantes. Se propone un nivel adicional a los 4 ya existentes, justificado en la necesidad de fortalecer y ampliar las competencias desarrolladas en esta asignatura por su importancia dentro del plan de estudios. En este sentido y teniendo en cuenta los énfasis vigentes dentro de la Licenciatura en Música: Instrumento, Dirección de conjuntos instrumentales y Dirección Coral, se plantea que el nivel 5 de la asignatura de Piano Complementario se trabaje con cada uno de los estudiantes de acuerdo al énfasis que planeen seleccionar.

El número de créditos pasa de uno a dos, debido a que el piano requiere el desarrollo de habilidades motoras, rítmicas, de lectura en dos pentagramas y de interpretación, lo cual implica un alto grado de compromiso y de trabajo individual por parte de los estudiantes. Se pretende desarrollar una clase en la que la interacción entre los estudiantes del mismo nivel promueva el aprendizaje colaborativo. Por lo anterior se considera que tres es un número apropiado de estudiantes para desarrollar la clase con una intensidad de una hora. En cuanto a las definición de la asignatura, el piano complementario se identifica como una herramienta musical y pedagógica, que por las posibilidades que ofrece de ver conceptos teóricos en apariencia abstractos y de adaptar casi cualquier tipo de música 
para este instrumento, se convierte en un apoyo fundamental de las asignaturas que hacen parte de la aprendizaje musical en todos los niveles de formación.

El objetivo general se centra en promover el aprendizaje del Piano Complementario como una herramienta de apoyo de los procesos de formación musical y pedagógica de los estudiantes de la Licenciatura en Música de la UPTC.

Lo anterior, implica, el desarrollar gradual y sistemáticamente procesos fluidos de lectura musical, transporte, armonización, improvisación y acompañamiento en el piano, estimular la sensibilidad del estudiante para que sea capaz de llegar a expresarse y comunicarse efectiva y estéticamente a través de la música y fomentar en los estudiantes el uso del piano como parte fundamental de su labor pedagógica como futuro Licenciado en Música.

Debido a que la investigación en la asignatura de Piano Complementario no se evidencia en los contenidos actuales, se promoverá en esta propuesta, a partir de la escucha crítica de repertorios propios del instrumento en diferentes versiones, así como la lectura de textos relacionados con la ejecución instrumental y con el repertorio asignado. Es indispensable que el estudiante como futuro músico-educador proponga soluciones creativas debidamente argumentadas a los problemas encontrados durante su proceso de aprendizaje instrumental.

Se sugiere desarrollar unas unidades temáticas similares a lo largo de los cuatro primeros niveles que aumentarán progresivamente de dificultad a medida que el estudiante avanza en su propio proceso. En este sentido se propone 1) Técnica, 2) Patrones de acompañamiento, 3) Armonización de melodías, 4) Montaje de canciones infantiles, 5) Interpretación de obras pianísticas y por último 6) Ensambles, donde se incluirán temas populares, de diversos géneros, entre los cuales está la música colombiana. Teniendo en cuenta que el nivel cinco de Piano Complementario se cursará de acuerdo al énfasis que vaya a seleccionar cada estudiante, se mantendrán dos unidades temáticas comunes a todos los énfasis y las demás se desarrollarán de manera específica para cada uno de ellos, así:

Énfasis de Instrumento Principal:1) Patrones de acompañamiento (Común a todos los énfasis), 2) Interpretación de repertorio pianístico (Común a todos los énfasis), 3) Música de cámara, 4) Lectura a primera vista.

Énfasis de Dirección Coral y Dirección de Agrupaciones:1) Patrones de acompañamiento (Común a todos los énfasis, 2) Interpretación de repertorio pianístico (Común a todos los énfasis), 3) Reducciones orquestales, 4) Lectura en 4 claves.

Al respecto de la metodología, si bien los procesos de aprendizaje de un instrumento musical son individuales y con ritmos diferentes de apropiación, en general puede plantearse un modelo basado en el enfoque constructivista, el aprendizaje significativo (Ausbel, 1976), el pensamiento crítico, el aprendizaje colaborativo (Quiñones, 2005) y recepción verbal (Rusinek, 2004). La pregunta será el fundamento de las explicaciones por parte del docente, con el propósito indagar en los conocimientos previos y fomentar el aprendizaje por descubrimiento. Los ejercicios pueden ser elegidos por los estudiantes con asesoría del docente que mediará entre los gustos musicales y la zona de desarrollo próximo (Piaget). Para garantizar que el aprendizaje sea interiorizado y entendido, se hará permanentemente transferencia de conocimientos a contextos variados.

La evaluación es establecida en sus generalidades por la institución en dos momentos, primer $50 \%$ y segundo $50 \%$. Sin embargo se propone que se siga trabajando con la ficha de seguimiento donde se evalúa permanentemente el proceso individual del estudiante y los resultados.

La bibliografía se plantea de manera general para todos los semestres, ya que cada método presenta diferentes niveles de dificultad que se pueden adaptar al desarrollo de la clase y a las particularidades de cada estudiante.

En el ámbito territorial, es importante que los contenidos planteados estén articulados con las necesidades sociales y culturales de la región y el país. Deben ser flexibles en cuanto se adapten a la idiosincrasia de los individuos y permitan diversidad de pensamientos, intereses, tendencias y modelos educativos.

El segundo ámbito, el económico, se relaciona 
directamente con el perfil laboral del egresado y su preparación para ser agente emprendedor de nuevas propuestas en el ámbito cultural y educativo de la región. En cuanto a lo político, la propuesta curricular derivada de este proyecto de investigación debe apuntar a la formación de personas que trabajen en equipo y que entiendan las dinámicas de liderazgo. Por último en el ámbito socio cultural y debido a que la investigación se relaciona con un programa humanístico y de la disciplina musical, el diseño y propuesta curricular debe generar gran impacto en cuanto a la identidad cultural y la transformación del pensamiento colectivo respecto a la concepción sobre el arte en la sociedad y sobre la importancia de la cultura en el desarrollo de una nación

\section{Conclusiones.}

La comunidad académica en general que integra la Licenciatura en Música de la UPTC (estudiantes, docentes y egresados del Programa) resaltan la gran importancia que el Piano Complementario tiene para los futuros Licenciados en Música. Es necesario que dicha importancia se refleje también en el lugar que la asignatura ocupa dentro del plan de estudios.

Las reformas curriculares que se hagan al plan de estudios de la Licenciatura en Música de la UPTC solo tendrán pertinencia y responderán a las necesidades del medio en la medida que involucren en su realización a la mayor parte posible de integrantes de la comunidad que integra el programa. (Estudiantes, docentes, egresados)

La opinión de los egresados del programa tiene un papel fundamental a la hora de conocer de manera directa las reales necesidades del medio en el que se desenvuelven. Es por ello que para la elaboración misma de los contenidos temáticos de las diversas asignaturas que hacen parte del currículo, se debería contar con su punto de vista.

En los contenidos curriculares de Piano Complementario de los programas de Licenciatura en Música del país que fueron analizados en este proyecto se puede apreciar que existen inquietudes comunes, en lo que tiene que ver con el tipo de enfoque que debe primar en la asignatura. Se observan además problemáticas comunes como el bajo número de crédito de la asignatura y que son muy pocos los niveles que deben cursar los estudiantes.

Dadas las problemáticas e inquietudes comunes a varios programas de Licenciatura en Música del país respecto al Piano Complementario, es pertinente comenzar a establecer un mayor diálogo entre los docentes de dicha asignatura en Colombia. Esto a futuro sin duda puede ayudar a encontrar soluciones conjuntas a las problemáticas encontradas y a la realización de proyectos conjuntos que redunden en un beneficio para estudiantes y docentes de la asignatura.

Si bien durante muchos años la formación musical se ha desarrollado siguiendo el modelo conservatorio heredado de la tradición de la música académica europea, manteniéndose de alguna forma al margen de las innovaciones didácticas que proponen diversas corrientes de la pedagogía general, la inserción de la enseñanza musical dentro de las Universidades exige cada vez más que dichas corrientes sean tenidas en cuenta a la hora de plantear los diversos contenidos curriculares de las asignaturas.

Si bien la asignatura de Piano Complementario es común a la gran mayoría de programas de Licenciatura en Música del país, la investigación permitió determinar con certeza particularidades del medio en el que se desenvuelven los egresados de la Licenciatura en Música de la UPTC, así como obtener una valiosa información sobre sus inquietudes respecto a la asignatura de Piano Complementario que pueden y deben tenerse en cuenta en una futura reestructuración de la misma.

El análisis sobre el desarrollo histórico que ha tenido el Piano Complementario en la Licenciatura en Música de la UPTC refleja que la asignatura no ha estado del todo articulada con las otras que integran el currículo. Por lo tanto y con el fin de que cumpla su función de herramienta de apoyo a los procesos de formación de los estudiantes, se hace necesario un mayor trabajo interdisciplinar con las demás asignaturas que integran el currículo.

Dada la gran importancia que la asignatura de Piano Complementario tiene para los futuros Licenciados en Música, es posible proyectar esta asignatura como un verdadero eje integrador 
que articula los diversos contenidos comunes de las asignaturas teóricas y pedagógicas en los procesos de formación musical y pedagógica de los estudiantes. Expresa proactivamente lo desarrollado y encontrado. Se puede presentar una síntesis de propuesta para enriquecer la solución encontrada a la problemática planteada.

\section{Referencias}

Ausubel, D. P., Novak, J. D., \& Hanesian, H. (1976). Psicología educativa: un punto de vista cognoscitivo (Vol. 3). México: Trillas.

Bueno, J. (2011). Diseño curricular para la cátedra de piano complementario en los conservatorios de música del Ecuador, una propuesta metodológica.(Tesis de Maestría). Universidad de Cuenca. Cuenca.

Bravo, E. (2009). Enseñanza en grupo del piano para estudiantes de otras especialidades musicales. (Tesis de Maestría). Universidad Nacional de Colombia. Bogotá.

Boardman, E. (1989). Dimensions of musical thinking. R\&L Education. Procesos de pensamiento Musical (Traducción Isabel Martinez)

Carrillo Siles, Beatriz (2009). Importancia del currículo oculto en el proceso enseñanza-aprendizaje. Revista digital Innovación y experiencias educativas. España.

Carretero, M. (2000). Constructivismo y educación. Editorial Progreso.

Davidson, L., \& Scripp, L. (1992). Surveying the coordinates of cognitive skills in music. In Handbook of Research on Music Teaching and Learning: a project of the Music Educators National Conference (pp. 392-413).

Delval, J. (2001) Hoy todos son constructivistas. Revista Educere, vol. 5, núm. 15, (ISSN: 13164910). Universidad de los Andes. Mérida, Venezuela pp. 353-359

Echeverri, H. (2014). Manual de armonía aplicada al teclado. (Tesis de maestría). Universidad Eafit. Medellín.

Garvin, M. (2011). Guía metodológica para la enseñanza de algunas piezas para piano del maes- tro Luis Carlos Figueroa. (Tesis de maestría). Universidad Nacional de Colombia. Bogotá.

Gellatly, A., \& Wald, M. (1986). La inteligencia hábil: el desarrollo de las capacidades cognitivas. Ed. Aique. Buenos Aires. Argentina

Mayo, I. C., \& Vargas, G. F. (2010). Del currículum musical prescrito al currículum musical práctico en el aula de educación primaria. Bordón. Revista de pedagogía, 62(2), 109-126.

Medina, L., \& Guzmán, L. (2011). Innovación curricular en las Instituciones de Educación Superior. Ed. ANUIES. México.

Murillo, J \& Martínez, C. (2010). Métodos de investigación educativa en educación especial. Recuperado en https: / / www.uam.es / personal_ pdi / stmaria/jmurillo/InvestigacionEE / Presentaciones/Curso_10/I_Etnografica_Trabajo.pdf

Quiñones, M. Ñ. (2005). El rol del maestro en un esquema pedagógico constructivista. Publicaciones sistema Universitario SUAGM: http:/ / bibliotecavirtualut. suagm. edu/Publicaciones.

Regelski, T. (1980) Principios y problemas de la educación musical. Ed. Diana. Mexico.

Rusinek, G. (2004). Aprendizaje musical significativo. Revista Electrónica Complutense de Investigación en Educación Musical-RECIEM, 1, 5.

Sampieri, R. (et al.). (2006). Metodología de la investigación. México D.F., México: Ed. Mc Graw Hill.

Silva, P. H. (2006). El reto de la transformación curricular. Revista Iberoamericana de educación, 40(3), 5.Ed. Organización de Estados Iberoamericanos para la Educación, la Ciencia y la Cultura (OEI). Cuba

Tuñez , M. \& Zabala, M. (2007) ¿Cuáles son hoy los puntos de partida en la enseñanza de un instrumento? Actas de la VI reunión de SACCOM. Pp.47-56

Universidad Pedagógica y Tecnológica de Colombia. (2009) Proyecto Académico Educativo de la Licenciatura en Música. Aprobado mediante Resolución del Consejo Académico No. 065 del 14 de Diciembre de 2009 


\section{Bibliografía}

Castro González, S. (2013). El Bossa Nova y el cifrado de la armonía popular: aportes para la práctica del análisis armónico.

Czerny, C., \& Sanders, E. (1956). Recollections from my life. The Musical Quarterly, 42(3), 302317.

Echeverri Pineda, H. H. (2014). Manual de armonía aplicada al teclado." Una propuesta didáctica, basada en la armonización y la improvisación para la enseñanza del piano complementario en universidades colombianas".

Friedman, D. P., \& Felleisen, M. (1996). The Little Schemer. MIT Press.

Hoover, M. (2010). A guide to the Latin American art song repertoire: an annotated catalog of twentieth-century art songs for voice and piano. Indiana University Press.
Levine, M. (2011). The jazz piano book. “ O’Reilly Media, Inc.".

Molina, E. (1999). Piano complementario 1: un nuevo modo de acercarse al piano. Real Musical.

Moreno Espinal, J. M. (2007). Aires folclóricos al piano: composiciones para piano con base en el folclor colombiano (Doctoral dissertation, Especialización en Artes).

Morocho Morocho, O. (2012). Método de acompañamiento de piano de música popular para jóvenes.

Peterson, O., \& Brown, R. (1965). Ray Brown presents Oscar Peterson complete jazz for the young pianist: exercises, minuets, etudes, pieces. Hansen.

Taylor, B. (1983). Jazz piano: A jazz history. WCB/McGraw-Hill. 